\title{
Errors in the Series-Parallel Buildup of Four-Terminal Resistors
}

\author{
Chester H. Page
}

(April 20, 1965)

\begin{abstract}
The use of $n$ equal resistors (a) in series and (b) in parallel provides an $n^{2}: 1$ ratio of potentially high accuracy. Such devices are important for extending the use of the national one-ohm standard to the hundred-ohm, and thence to the 10000 -ohm, level.

Formulas are derived for the error in the ratio, expressed (a) in terms of design tolerance, and (b) in terms of first-order residual misadjustments which combine to yield the secondorder error of the ratio.

It seems feasible to construct a 1:100 ohm buildup device with a ratio uncertainty of less than 1 in $10^{8}$.
\end{abstract}

The use of $n$ equal resistors (a) in series and (b) in parallel provides an $n^{2}: 1$ ratio of potentially high accuracy. Such devices are important for extending the use of the national $1-\Omega$ standard to the $100-\Omega$, and thence to the $10000-\Omega$ level.

The first stage of this step-up involves the use of ten 10-ohm resistors, which must be four-terminal resistors to avoid large errors due to the connecting networks.

Hamon ${ }^{1}$ has described an arrangement of fourterminal resistors permanently connected in series, and convertible to a parallel connection by adding jumpers. In that paper, he shows the use of compensating resistors in the potential leads to eliminate errors introduced by the added connections.

Compensated lead "fans" may be used for the potential terminals of the paralleled resistors, for the current terminals, or for both. The aim of the present paper is to present a complete analysis of the general case, and formulas for the errors introduced by imperfectly compensated fans.

1 B. V. Hamon, A $100 \Omega$ build-up resistor for the calibration of standard resistors, J. Sci. Instr. 31, 450-453 (Dec. 1954).
We consider four-terminal resistors connected in series by means of "tetrahedral" junctions, having. the equivalent circuit shown in figure 1. Each junction supplies current and potential leads. This array can be converted to a parallel connection by adding four "terminal fans," as in figure 2. For analysis, the junction resistances can be considered as absorbed in the fan-conductor resistances. The problem is to make the four-terminal resistance of the combination precisely equal to $R / n$. If the resistances of the various arms of the current fan are adjusted to make each main resistor $(R)$ carry identical current, the corresponding voltage drops will be identical and there will be no circulating current in the potential fans, and the potential across $V_{1}-V_{2}$ will be independent of the resistances of the potential fans, and equal to $I R / n$. This requires that each arm of the current fan on the left have the same resistance, say $r$, except for the top and bottom arms, each of which feeds only one main resistor. These end arms must have the resistance $2 r$. For the right-hand current fan, each arm must have the same resistance, say $r^{\prime}$. The reciprocity theorem leads to the conclusion that if we use these compensated fans as potential fans, the four-terminal

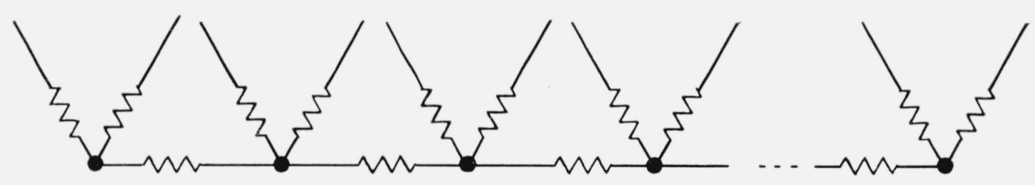

Figure 1 


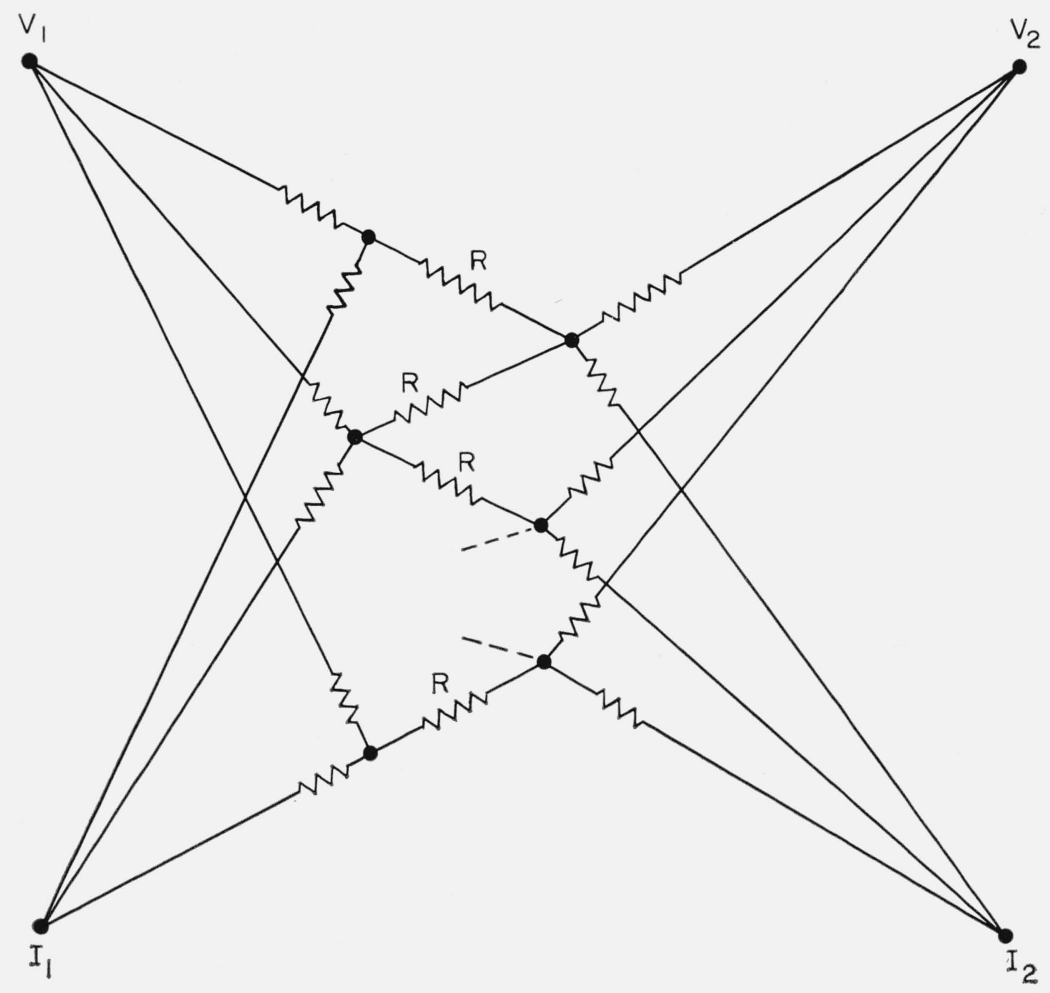

Figure 2

resistance would be $R / n$, independent of the currentfan resistances. Since the potential fans can tolerate larger resistance than can the current fans, the compensation is usually made in the potential fans; resistances of several tenths of an ohm can be added to the arms, allowing adjustment to reasonable accuracy.

Making either set of fans perfect yields zero error. This suggests that the overall error is in the nature of a product of fan errors, and suggests the possibility of reducing the effect of residual potential fan errors by making at least a rough adjustment of the current fan. For analysis, we consider four perfectly compensated fans as a nominal condition, with arbitrary maladjustments allowed in each arm. In addition each main resistor is allowed a departure from nominal.

The complete circuit to be analyzed is shown in figure 3 , where the labels indicate conductance rather than resistance. Capital letters indicate "average" values, i.e.,

$$
\sum a_{i}=\sum b_{i}=\sum c_{i}=\sum d_{i}=\sum g_{i}=0 .
$$

Considering terminal $G$ as the "ground" terminal, we have a network possessing three external nodes, and $(n+1)$ internal nodes. A complete description of the network requires $3+(n+1)$ simultaneous equations, and we must eliminate the last $n+1$.
If we impress currents $I_{\mathrm{I}}, I_{\mathrm{II}}, I_{\mathrm{III}}$ in to the external nodes, and $I_{1}, I_{2} \ldots I_{n+1}$ into the internal nodes, the voltages on these nodes are implied by the simultaneous equations:

$$
\begin{array}{ll}
I_{\mathrm{I}}=Y_{\mathrm{II}} V_{\mathrm{I}}+Y_{\mathrm{III}} V_{\mathrm{II}}++Y_{\mathrm{II}} V_{1}+ & +Y_{\mathrm{I} n+1} V_{n+1} \\
I_{\mathrm{II}}=Y_{\mathrm{III} I} V_{\mathrm{I}}+ & +Y_{\mathrm{II} n+1} V_{n+1} \\
\vdots & \\
I_{n+1}=Y_{n+1 \mathrm{I}} V_{\mathrm{I}}+ & +Y_{n+1 n+1} V_{n+1}
\end{array}
$$

These equations are conveniently symbolized by the matric equation

$$
I=Y V
$$

where $I$ and $V$ are column vectors (each having $n+4$ components), and $Y$ is an $(n+4) \times(n+4)$ square matrix.

Consider $I_{\mathrm{I}}, I_{\mathrm{II}}$, and $I_{\mathrm{III}}$ as components of an "external" current vector, $I_{e}$, and $\bar{I}_{1} \ldots I_{n+1}$ as components of an "internal" current vector, $I_{i}$; similarly consider "external" and "internal" unknown voltage vectors, $V_{e}$ and $V_{i}$. Grouping the terms appropriately:

$$
\begin{aligned}
& I_{e}=\alpha V_{e}+\beta V_{i} \\
& I_{i}=\tilde{\beta} V_{e}+\gamma V_{i}
\end{aligned}
$$




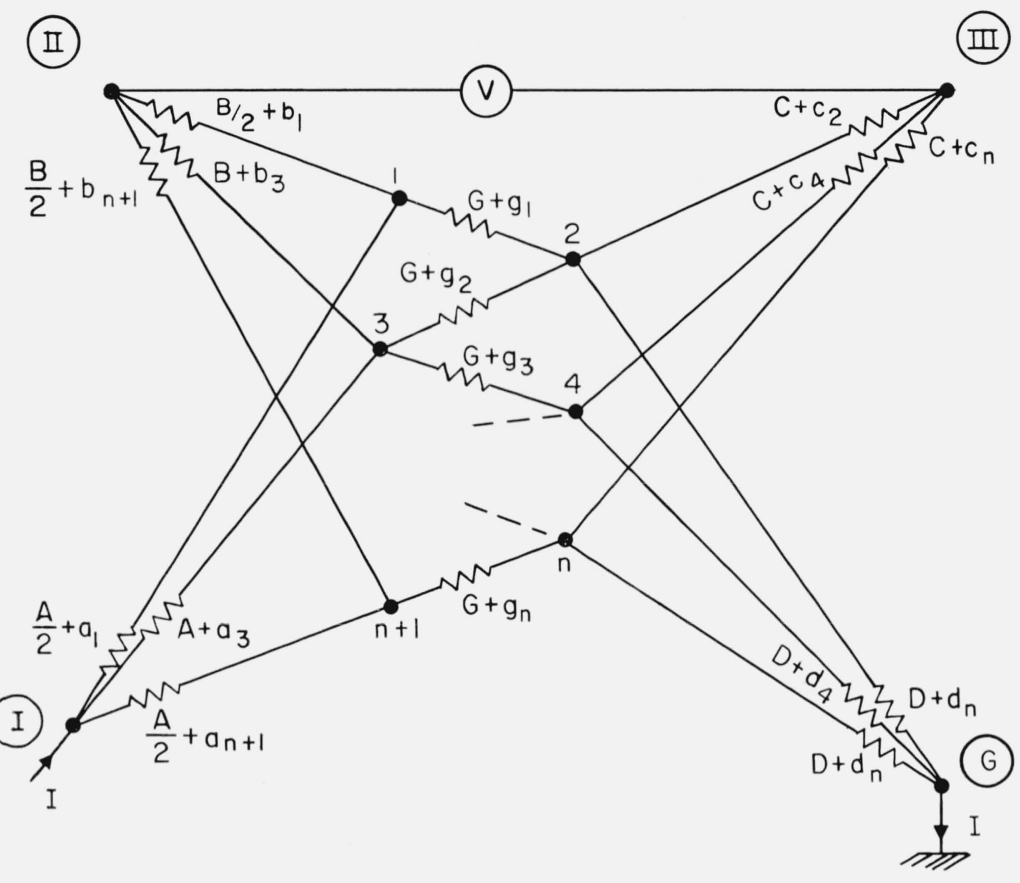

Figure 3

where $\alpha$ is a $3 \times 3$ matrix, $\beta$ is a $3 \times(n+1)$ matrix, $\tilde{\beta}$ is its transpose, and $\gamma$ is an $(n+1) \times(n+1)$ matrix. In other words, the matrix is partitioned:

$$
I=\left(\begin{array}{ll}
\alpha & \beta \\
\tilde{\beta} & \gamma
\end{array}\right) V .
$$

Since we are interested in knowing the external voltage with no impressed internal current, we must eliminate $V_{i}$ from

$$
\begin{gathered}
I_{e}=\alpha V_{e}+\beta V_{i} \\
0=\tilde{\beta} V_{e}+\gamma V_{i}
\end{gathered}
$$

finding

$$
\begin{gathered}
I_{e}=\left(\alpha-\beta \gamma^{-1} \tilde{\beta}\right) V_{e} \\
V_{e}=\left(\alpha-\beta \gamma^{-1} \tilde{\beta}\right)^{-1} I_{e} .
\end{gathered}
$$

This last equation can be written as

$$
\left(\begin{array}{l}
V_{\mathrm{I}} \\
V_{\mathrm{II}} \\
V_{\mathrm{III}}
\end{array}\right)=\left(\begin{array}{lll}
z_{11} & z_{12} & z_{13} \\
z_{21} & z_{22} & z_{23} \\
z_{31} & z_{32} & z_{33}
\end{array}\right) \quad\left(\begin{array}{c}
I_{\mathrm{I}} \\
I_{\mathrm{II}} \\
I_{\mathrm{III}}
\end{array}\right) .
$$

The required four-terminal resistance is the transfer resistance $\left(V_{\mathrm{II}}-V_{\mathrm{III}}\right) / I_{\mathrm{I}}$ under the condition $I_{\mathrm{II}}=$ $I_{\mathrm{III}}=0$, hence

$$
R=\left(z_{21}-z_{31}\right) \equiv(0,1,-1) z\left(\begin{array}{l}
1 \\
0 \\
0
\end{array}\right) .
$$

The problem is to carry out all the indicated algebraic manipulations, with all resistors subject to arbitrary tolerances. This will be done in an appendix, using the following strategy.

First, we carry out the manipulations required for $\left(\alpha-\beta \gamma^{-1} \tilde{\beta}\right)^{-1}$ assuming the nominal values of the network elements; then, assuming that the departures from nominal are small, find the resulting correction to the nominal $R_{0}\left(=\frac{1}{n G}\right)$ as a series in powers of the perturbations. This yields a formula for the fractional error as a sum of terms of the type $g_{i} g_{j} ; g_{i} a_{i}, g_{i} b_{i}$, etc.; $a_{i} b_{i}, c_{i} d_{i}$. The terms involving the $g_{i}$ (tolerances in the resistors of the series-parallel set) are ordinarily negligible compared with the pure measurement-network errors. These latter give (from eq (A38)):

Error $=\frac{4}{n} \frac{G}{A+B}\left(2 \frac{a_{1}}{A} \frac{b_{1}}{B}+\frac{a_{3}}{A} \frac{b_{3}}{B}\right.$

$$
\begin{gathered}
\left.+\frac{a_{5}}{A} \frac{b_{5}}{B}+\ldots+2 \frac{a_{n+1}}{A} \frac{b_{n+1}}{B}\right) \\
+\frac{4}{n} \frac{G}{C+D}\left(\frac{c_{2}}{C} \frac{d_{2}}{D}+\frac{c_{4}}{C} \frac{d_{4}}{D}+\ldots+\frac{c_{n}}{C} \frac{d_{n}}{D}\right) .
\end{gathered}
$$


Since $\Sigma a_{i}=0$, etc., this error arises from accidental correlation between errors of fan connections to common points. The worst case is where half the $a_{i}$ have maximum positive error, the other half having maximum negative error-and where these pair up with the same situation among the $b_{i}$. Normal design would have $D=A, B=C$, and $A>B$. Now let the maximum fractional error in the voltage fans be $\boldsymbol{\epsilon}_{v}$; in the current fans, $\boldsymbol{\epsilon}_{i}$. The resulting extreme error is $4 \frac{G}{A} \epsilon_{v} \epsilon_{i}$. Reasonable estimates are $\frac{1}{G}=10 \Omega, \frac{1}{A}=1 \mathrm{~m} \Omega, \epsilon_{i}=10^{-1}, \epsilon_{v}=10^{-3}$, yielding a maximum error in the series-parallel ratio of $4 \times 10^{-8}$. Fractional errors of $10^{-5}$ in the main resistors make the $g_{i}$ contributions completely negligible.

The above computation is for maximum error. Random pairing of the fan errors gives a much smaller expected error.

The next problem is to adjust the device after it has been constructed, and to evaluate the residual error. We note that if the current fans are perfect, there will be no voltage across any arm of the potential fans, hence any voltage across an arm of a potential fan is a measure of current-fan errors.

The potential fans have relatively high resistance, so are conveniently "trimmed" on a resistance bridge. To adjust the current fans, we can connect a microvoltmeter across each potential fan resistor in turn. This essentially measures the deviation of the potentials on the internal nodes, from their average. The current-fan arms can be filed to reduce these voltages to approximately zero.

In the analysis, we have treated the tetrahedral junctions as though their centers were available terminals. Any adjustment procedure must take cognizance of the fact that these terminals are fictitious; any physical connection is separated from the tetrahedron center by a low resistance. This could cause errors in direct measurement of currentfan resistance. The proposed scheme of measuring potential-fan resistances and voltage drops avoids this difficulty, since the potential fans are loaded with additional resistance.

In appendix $A$, we evaluate these residual adjustment errors quantitatively. We find to first order that the voltage across the arm $B+b_{3}$ is given by

$$
I X_{3}=\frac{2}{n(A+B)} \frac{a_{3}}{A} I_{i}
$$

and similarly the voltage across $A+a_{3}$, for current $I$ into terminal (II) and out of terminal (III), is

$$
I W_{3}=\frac{2}{n(A+B)} \frac{b_{3}}{B} I
$$

Comparison with eq (9) yields

$$
\begin{aligned}
& \text { Error }=n G\{(A+B) \\
& \begin{aligned}
\left(\frac{X_{1} W_{1}}{4}+X_{3} W_{3}+X_{5} W_{5}+\ldots+\frac{X_{n+1} W_{n+1}}{4}\right) \\
\left.\quad+(C+D)\left(X_{2} W_{2}+X_{4} W_{4}+\ldots+X_{n} W_{n}\right)\right\} .
\end{aligned}
\end{aligned}
$$

For a test current of $1 \mathrm{~A}$, the fan tolerances assumed before yield voltages of the order

with

$$
\begin{aligned}
& I X \sim 2 \times 10^{-5} \mathrm{~V} \\
& I W \sim 2 \times 10^{-7} \mathrm{~V}
\end{aligned}
$$

$$
n G A \sim 10^{3} .
$$

Note that the larger voltage is generated by the current-fan error (under the assumed construction tolerances); this should make it feasible to adjust the current fan to the next order of magnitude.

The most sensitive set of measurements for evaluating the residual error is probably the set of residual adjustment errors; i.e., the residual potential-fan resistance differences and voltage drops. The appropriate formula is (from eq (A34)):

Residual error $=2 G\left\{X_{1} b_{1} / B+X_{3} b_{3} / B \ldots\right.$

$$
\left.+X_{n+1} b_{n+1} / B-X_{2} c_{2} / C-X_{4} c_{4} / C-\ldots-X_{n} c_{n} / C\right\}
$$

where positive $X_{i}$ is associated with node $i$ positive with respect to the appropriate potential terminal.

Note that the measured error quantities are firstorder effects in the network tolerances; the computed residual error is a second-order product of these terms, so can be reasonably accurately evaluated by this procedure.

There are, of course, additional errors not attributable to the current and potential fans. The first of these arises from the fact that the series resistance is not $n / G$, but is

$$
R_{s}=\sum \frac{1}{G+g_{i}} \doteq \frac{n}{G}\left(1+\frac{1}{n} \sum g_{i}^{2} / G^{2}\right)
$$

yielding a fractional error of only $10^{-10}$ for $g_{i} / G \sim 10^{-5}$. Another source of error lies in the imperfections of the tetrahedral junctions. Under conditions of suitable design and proper adjustment procedure, these junctions can be balanced to transfer resistances of less than $10^{-8} \Omega$. In a string of $10-\Omega$ resistors, this is then a potential source of error of the order of $10^{-9}$.

It seems feasible, therefore, to construct a 1:100 $\Omega$ buildup device with a ratio uncertainty of less than 1 in $10^{8}$. 


\section{Appendix A}

The submatrices of equation (4) are

$\alpha=\left(\begin{array}{ccc}\frac{A}{2}+a_{1}+A+a_{3}+\ldots & 0 & 0 \\ 0 & \frac{B}{2}+b_{1}+B+b_{3}+\ldots & 0 \\ 0 & 0 & C+c_{2}+C+c_{4}+\ldots\end{array}\right)$

$$
=\left(\begin{array}{ccc}
\frac{n A}{2} & 0 & 0 \\
0 & \frac{n B}{2} & 0 \\
0 & 0 & \frac{n C}{2}
\end{array}\right)=\frac{n}{2}\left(\begin{array}{ccc}
A & 0 & 0 \\
0 & B & 0 \\
0 & 0 & C
\end{array}\right)
$$

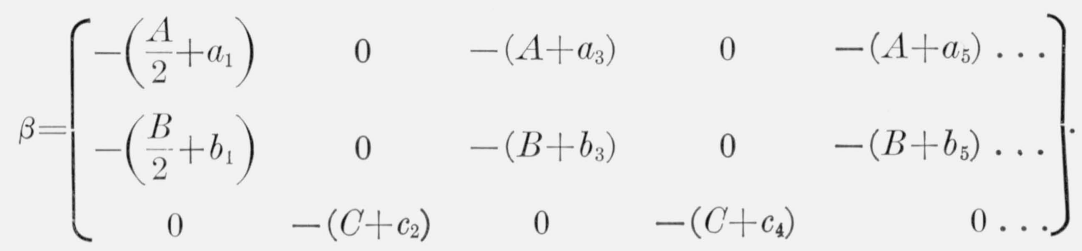

$$
\gamma=\left(\begin{array}{ccc}
G+g_{1}+\frac{A}{2}+a_{1}+\frac{B}{2}+b_{1} & -\left(G+g_{1}\right) & 0 \ldots \\
-\left(G+g_{1}\right) & 2 G+g_{1}+g_{2}+C+c_{2}+D+d_{2} & -\left(G+g_{2}\right) \ldots \\
0 & -\left(G+g_{2}\right) & 2 G+g_{2}+g_{3}+A+a_{3}+B+b_{3} \ldots \\
\vdots & \vdots & \vdots
\end{array}\right) .
$$

It is convenient to decompose these matrices into sums of nominal values and perturbations:

Let

where

$$
\begin{aligned}
& \beta \equiv \beta_{0}+\beta_{1} \\
& \gamma \equiv \Gamma+\gamma_{0}+\gamma_{1}+\gamma_{2}
\end{aligned}
$$

$$
\beta_{0}=\left(\begin{array}{cccccc}
-A / 2 & 0 & -A & 0 & -A & \ldots \\
-B / 2 & 0 & -B & 0 & -B & \ldots \\
0 & -C & 0 & -C & 0 & \ldots
\end{array}\right)
$$

$$
\beta_{1}=\left(\begin{array}{ccccc}
-a_{1} & 0 & -a_{3} & 0 & \ldots \\
-b_{1} & 0 & -b_{3} & 0 & \ldots \\
0 & -c_{2} & 0 & -c_{4} & \ldots
\end{array}\right)
$$

$$
\gamma_{0} \equiv G S
$$

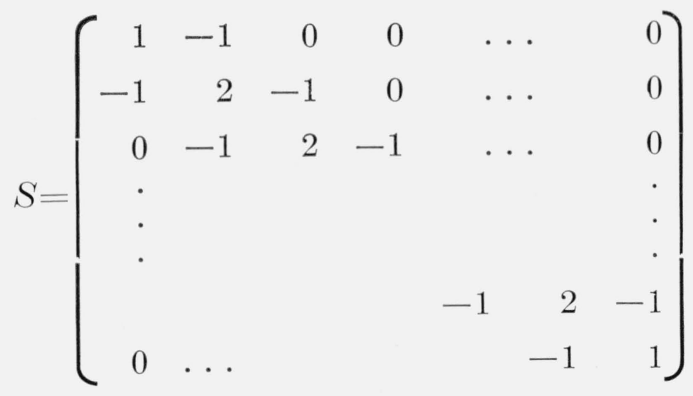




$$
\gamma_{1}=\left(\begin{array}{ccccc}
a_{1}+b_{1} & 0 & 0 & \ldots & 0 \\
0 & c_{2}+d_{2} & 0 & \ldots & 0 \\
0 & 0 & a_{3}+b_{3} & \ldots & 0 \\
\cdot & & & & \cdot \\
\cdot & & & & \cdot \\
\cdot & & & & a_{n+1}+b_{n+1}
\end{array}\right)
$$

$\gamma_{2}=\left(\begin{array}{cccccc}g_{1} & -g_{1} & 0 & 0 & \ldots & 0 \\ -g_{1} & g_{1}+g_{2} & -g_{2} & 0 & \ldots & 0 \\ 0 & -g_{2} & g_{2}+g_{3} & -g_{3} & \ldots & 0 \\ \cdot & & & & & \cdot \\ \cdot & & & & & \cdot \\ \cdot & & & & g_{n-1}+g_{n} & -g_{n} \\ & & & & -g_{n} & g_{n}\end{array}\right)$

(A10)

We write the admittance matrix as the sum of a nominal value and a perturbation:

$$
\begin{aligned}
Y & =\left(\begin{array}{cc}
\alpha & \beta_{0} \\
\tilde{\beta}_{0} & \Gamma+\gamma_{0}
\end{array}\right)+\left(\begin{array}{cc}
0 & \beta_{1} \\
\tilde{\beta} & \gamma_{1}+\gamma_{2}
\end{array}\right) \\
& \equiv Y_{0}\left(\begin{array}{cc}
0 & \beta_{1} \\
\tilde{\beta}_{1} & \epsilon
\end{array}\right) .
\end{aligned}
$$

Let

$$
Y_{0}^{-1} \equiv\left(\begin{array}{cc}
Z_{0} & Z_{1} \\
\tilde{Z}_{1} & Z_{2}
\end{array}\right)
$$

where

$$
\begin{aligned}
& Z_{0}=\left(\alpha-\beta_{0}\left(\Gamma+\gamma_{0}\right)^{-1} \tilde{\beta}_{0}\right)^{-1} \\
& Z_{1}=-Z_{0} \beta_{0}\left(\Gamma+\gamma_{0}\right)^{-1} \\
& Z_{2}=\left(\Gamma+\gamma_{0}\right)^{-1}-\left(\Gamma+\gamma_{0}\right)^{-1} \tilde{\beta}_{0} Z_{1} .
\end{aligned}
$$

Now $\left(\Gamma+\gamma_{0}\right)^{-1}$ is not readily found, but the combination $\beta_{0}\left(\Gamma+\gamma_{0}\right)^{-1}$ is, from appendix $\mathrm{B}$ :

$\beta_{0}\left(\Gamma+\gamma_{0}\right)^{-1}=-[1+2 G(E+F)]^{-1}$

$$
\begin{aligned}
& \left\{2 \operatorname{GEF}\left(\begin{array}{lll}
A & A & A \\
B & B & B \\
C & C & C \ldots
\end{array}\right)\right. \\
& \left.+\left(\begin{array}{ccccc}
A E & 0 & A E & 0 & \ldots \\
B E & 0 & B E & 0 & \ldots \\
0 & C F & 0 & C F & \ldots
\end{array}\right)\right\}
\end{aligned}
$$

where

$$
E \equiv \frac{1}{A+B}, F \equiv \frac{1}{C+D}
$$

This yields

$$
\begin{aligned}
& Z_{0}=\frac{1}{n}\left(\begin{array}{ccc}
\frac{2}{A}+\frac{1}{G}+\frac{2}{D} & \frac{1}{G}+\frac{2}{D} & \frac{2}{D} \\
\frac{1}{G}+\frac{2}{D} & \frac{2}{B}+\frac{1}{G}+\frac{2}{D} & \frac{2}{D} \\
\frac{2}{D} & \frac{2}{D} & \frac{2}{C}+\frac{2}{D}
\end{array}\right)
\end{aligned}
$$

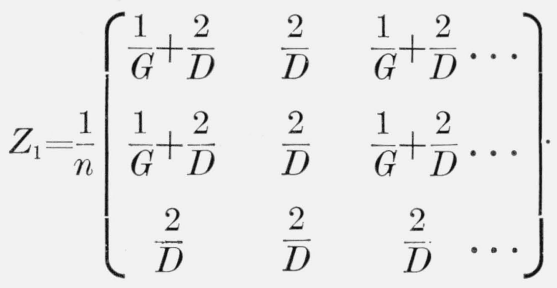

These matrices are readily verified by inspection of the circuit when the elements have their nominal values.

The matrix $Z_{2}$ involves the term $\left(\Gamma+\gamma_{0}\right)^{-1}$ standing by itself; we shall eventually need this term and shall use a series expansion for it.

The four-terminal resistance of interest is the transfer resistance expressed as $V_{\mathrm{II}}-V_{\mathrm{III}}$ for $I_{\mathrm{I}}=1$, all other input currents being zero:

$$
\left.R=\left[\begin{array}{lll}
0 & 1-1
\end{array}\right)\left(\begin{array}{lll}
0 & 0 \ldots 0
\end{array}\right] Y^{-1}\left[\begin{array}{l}
( \\
0 \\
0
\end{array}\right)\right]
$$

In (A16), we have partitioned the vectors into "external" and "internal" vectors, corresponding to the partitioning of $Y$. For the nominal case we find

$$
R_{0}=\left(\begin{array}{ll}
0 & 1-1
\end{array}\right) Z_{0}\left(\begin{array}{l}
1 \\
0 \\
0
\end{array}\right)=\frac{1}{n G} .
$$

We are also interested in the transfer resistances that are measured as voltage drops across the potentialfan arms:

$$
\left.X_{1}=\left[\begin{array}{llll}
(0-1 & 0
\end{array}\right)\left(\begin{array}{lll}
1 & 0 & 0 \ldots 0
\end{array}\right] Y^{-1}\left[\begin{array}{c}
1 \\
0 \\
0
\end{array}\right)\right]
$$




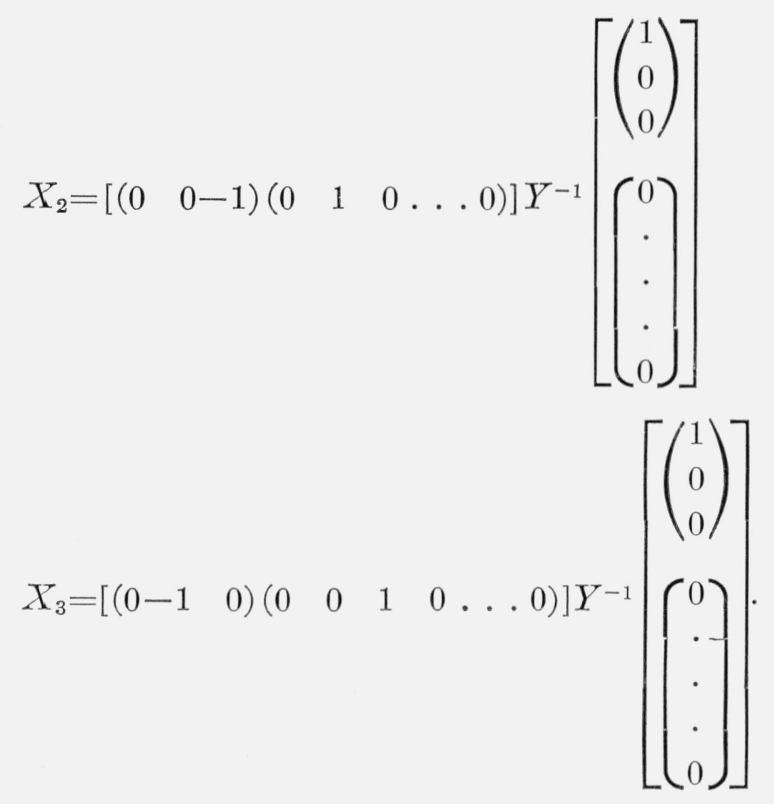

(A18)
All $X_{i}=0$ for the nominal case.

The perturbation from nominal is found by expanding $Y^{-1}$ in a series (as shown in appendix B for $\left.\left(\Gamma+\gamma_{0}\right)^{-1}\right)$ :

$$
\begin{aligned}
Y^{-1}= & Y_{0}^{-1}-Y_{0}^{-1}\left(\begin{array}{cc}
0 & \beta_{1} \\
\tilde{\beta}_{1} & \epsilon
\end{array}\right) Y_{0}^{-1} \\
& +Y_{0}^{-1}\left(\begin{array}{cc}
0 & \beta_{1} \\
\tilde{\beta}_{1} & \epsilon
\end{array}\right) Y_{0}^{-1}\left(\begin{array}{cc}
0 & \beta_{1} \\
\tilde{\beta}_{1} & \epsilon
\end{array}\right) Y_{0}^{-1}+\ldots
\end{aligned}
$$

to second order in the perturbation.

The algebra will be alleviated by noting some special properties of the matrices $\beta_{1}, \gamma_{1}$, and $\gamma_{2}$ :

$$
\begin{aligned}
& \left(\begin{array}{llll}
1 & 0 & 1 & 0 \ldots
\end{array}\right) \tilde{\beta}_{1}=\left(\begin{array}{lll}
0 & 0 & 0
\end{array}\right) \\
& \left(\begin{array}{llll}
0 & 1 & 0 & 1 \ldots
\end{array}\right) \tilde{\beta}_{1}=\left(\begin{array}{lll}
0 & 0 & 0
\end{array}\right)
\end{aligned}
$$

since $\Sigma a_{i}=\Sigma b_{i}=\Sigma c_{i}=0$

$$
\begin{aligned}
& \left(\begin{array}{lllll}
1 & 0 & 1 & 0 & \ldots
\end{array}\right) \gamma_{1}=\left(\begin{array}{lllll}
a_{1}+b_{1} & 0 & a_{3}+b_{3} & 0 & \ldots
\end{array}\right) \\
& \left(\begin{array}{llll}
0 & 1 & 0 & 1 \ldots
\end{array}\right) \gamma_{1}=\left(\begin{array}{llll}
0 & c_{2}+d_{2} & 0 & c_{4}+d_{4}
\end{array} \ldots\right) \\
& \left(\begin{array}{lllll}
1 & 0 & 1 & 0 & \ldots
\end{array}\right) \gamma_{2}=\left(\begin{array}{llll}
g_{1} & -\left(g_{1}+g_{2}\right) & \left(g_{2}+g_{3}\right) & \ldots
\end{array}\right) \\
& \left(\begin{array}{lllll}
0 & 1 & 0 & 1 \ldots
\end{array}\right) \gamma_{2}=\left(\begin{array}{llll}
-g_{1} & \left(g_{1}+g_{2}\right) & -\left(g_{2}+g_{3}\right) & \ldots
\end{array}\right) .
\end{aligned}
$$

Note that premultiplication of either $\gamma_{1}$ or $\gamma_{2}$ by either $\left(\begin{array}{llllll}1 & 0 & 1 & 0 & \ldots\end{array}\right)$ or $\left(\begin{array}{lllll}0 & 1 & 0 & 1 & \ldots\end{array}\right)$, followed by postmultiplication by either

$$
\left(\begin{array}{c}
1 \\
0 \\
1 \\
0 \\
\cdot \\
\cdot
\end{array}\right) \text { or }\left(\begin{array}{c}
0 \\
1 \\
0 \\
1 \\
\cdot \\
\cdot
\end{array}\right) \text { results in a null vector. }
$$

Thus from (A12b) and (A13) in conjunction with (A20) and (A21), we have the very useful relations:

$$
\begin{aligned}
Z_{1} \tilde{\beta}_{1} & =\mathbf{0} \\
Z_{1} \gamma_{1} \tilde{Z}_{1} & =\mathbf{0} \\
Z_{1} \gamma_{2} \tilde{Z}_{1} & =\mathbf{0} .
\end{aligned}
$$

These simplify the multiplications required by eq (A19):

$$
\begin{aligned}
Y_{0}^{-1}\left(\begin{array}{cc}
0 & \beta_{1} \\
\tilde{\beta}_{1} & \epsilon
\end{array}\right) & =\left(\begin{array}{cc}
Z_{0} & Z_{1} \\
\tilde{Z}_{1} & Z_{2}
\end{array}\right)\left(\begin{array}{cc}
0 & \beta_{1} \\
\tilde{\beta}_{1} & \epsilon
\end{array}\right)=\left(\begin{array}{cc}
0 & Z_{0} \beta_{1}+Z_{1} \epsilon \\
Z_{2} \tilde{\beta}_{1} & \tilde{Z}_{1} \beta_{1}+Z_{2} \epsilon
\end{array}\right) \\
& \equiv\left(\begin{array}{cc}
0 & M \\
Z_{2} \tilde{\beta}_{1} & \tilde{Z}_{1} \beta_{1}+Z_{2} \epsilon
\end{array}\right) .
\end{aligned}
$$

Note that

$$
M \tilde{Z}_{1}=Z_{0} \beta_{1} \tilde{Z}_{1}+Z_{1} \epsilon \tilde{Z}_{1}=\mathbf{0}, \quad Z_{1} \tilde{M}=\mathbf{0}
$$

so that

$$
Y_{0}^{-1}\left(\begin{array}{cc}
0 & \beta_{1} \\
\tilde{\beta}_{1} & \epsilon
\end{array}\right) Y_{0}^{-1}=\left(\begin{array}{cc}
0 & M Z_{2} \\
Z_{2} \tilde{M} & Z_{2} \tilde{\beta}_{1} Z_{1}+\tilde{Z}_{1} \beta_{1} Z_{2}+Z_{2} \epsilon Z_{2}
\end{array}\right)
$$

and finally

$Y_{0}^{-1}\left(\begin{array}{cc}0 & \beta_{1} \\ \tilde{\beta}_{1} & \epsilon\end{array}\right) Y_{0}^{-1}\left(\begin{array}{cc}0 & \beta_{1} \\ \tilde{\beta}_{1} & \epsilon\end{array}\right) Y_{0}^{-1}$ 


$$
=\left(\begin{array}{cc}
M z_{2} \tilde{M} & M z_{2}\left(\tilde{\beta}_{1} z_{1}+\epsilon z_{2}\right) \\
\left(z_{2} \epsilon+\tilde{z}_{1} \beta_{1}\right) z_{2} \tilde{M} & \text { etc. }
\end{array}\right) .
$$

Using these expressions in (A19) yields

$$
R=\frac{1}{n G}+\left(\begin{array}{ll}
0 & 1-1
\end{array}\right) M Z_{2} \tilde{M}\left(\begin{array}{l}
1 \\
0 \\
0
\end{array}\right)
$$

with no first order correction.

The $X_{i}$ have first order terms:

$$
\begin{gathered}
X_{1}=-\left(\begin{array}{lll}
1 & 0 & 0
\end{array}\right) Z_{2} \tilde{M}\left(\begin{array}{l}
1 \\
0 \\
0
\end{array}\right) \\
X_{2}=-\left(\begin{array}{lll}
0 & 1 & 0
\end{array}\right) Z_{2} \tilde{M}\left(\begin{array}{l}
1 \\
0 \\
0
\end{array}\right) \\
X_{3}=-\left(\begin{array}{llll}
0 & 0 & 1 & 0 \ldots
\end{array}\right) Z_{2} \tilde{M}\left(\begin{array}{l}
1 \\
0 \\
0
\end{array}\right)
\end{gathered}
$$

(A28)

etc.

These can be interpreted as the components of a vector, $X$ :

$$
X=-Z_{2} \tilde{M}\left(\begin{array}{l}
1 \\
0 \\
0
\end{array}\right)
$$

We can now write

$$
R=\frac{1}{n G}-\left(\begin{array}{ll}
0 & 1-1
\end{array}\right) M X
$$

as an alternate expression.

From (A12c) and (A24) we have

$$
Z_{2} \tilde{M}=\left(\Gamma+\gamma_{0}\right)^{-1} \tilde{M}
$$

so that

$$
R=\frac{1}{n G}+\left(\begin{array}{ll}
0 & 1-1
\end{array}\right) M\left(\Gamma+\gamma_{0}\right)^{-1} \tilde{M}\left(\begin{array}{l}
1 \\
0 \\
0
\end{array}\right) .
$$

Now

$\left(\begin{array}{l}0 \\ 1-1\end{array}\right) M \equiv\left(\begin{array}{ll}0 & 1-1\end{array}\right) Z_{0} \beta_{1}+\left(\begin{array}{ll}0 & 1-1\end{array}\right) Z_{1} \epsilon$

$$
\begin{aligned}
=\frac{1}{n}\left(\frac{1}{G}, \frac{2}{B}+\frac{1}{G},-\frac{2}{C}\right) \beta_{1} & \\
& +\frac{1}{n G}\left(\begin{array}{lllll}
1 & 0 & 1 & 0 \ldots)\left(\gamma_{1}+\gamma_{2}\right)
\end{array}\right. \\
= & \frac{1}{n}\left(\frac{g_{1}}{G}-\frac{2 b_{1}}{B},-\frac{g_{1}+g_{2}}{G}+\frac{2 c_{2}}{C},\right. \\
& \left.\frac{g_{2}+g_{3}}{G}-\frac{2 b_{3}}{B}, \ldots\right) . \quad(\mathrm{A} 33)
\end{aligned}
$$

Neglecting $g / G$ relative to $b / B$ and $c / C$, we have

$$
R \doteq \frac{1}{n G}+\frac{2}{n} \sum X_{2 i+1} b_{2 i+1} / B-\frac{2}{n} \sum X_{2 i} c_{2 i} / C
$$

for evaluation of error in terms of measured residuals.

To express $R$ in terms of circuit element tolerances, we need to express $X$ :

$$
\begin{aligned}
\tilde{X} & =-\left(\begin{array}{lll}
1 & 0 & 0
\end{array}\right) M\left(\Gamma+\gamma_{0}\right)^{-1} \\
& =-\left(\begin{array}{lll}
1 & 0 & 0
\end{array}\right)\left\{Z_{0} \beta_{1}+Z_{1}\left(\gamma_{1}+\gamma_{2}\right)\right\}\left(\Gamma+\gamma_{0}\right)^{-1}
\end{aligned}
$$

which becomes

$$
\begin{aligned}
\tilde{X}=\frac{1}{n}\left(\frac{2 a_{1}}{A}-\frac{g_{1}}{G},-\right. & \frac{2 d_{2}}{D}+\frac{g_{1}+g_{1}}{G}, \\
& \left.\frac{2 a_{3}}{A}-\frac{g_{2}+g_{3}}{G}, \ldots\right)\left(\Gamma+\gamma_{0}\right)^{-1} .
\end{aligned}
$$

We need $\left(\Gamma+\gamma_{0}\right)^{-1}$; we have

$\left(\Gamma+\gamma_{0}\right)^{-1} \equiv(\Gamma+G S)^{-1}=\Gamma^{-1}-G \Gamma^{-1} S \Gamma^{-1}$

Now

$$
+G^{2} \Gamma^{-1} S \Gamma^{-1} S \Gamma^{-1}-\ldots
$$

$$
\Gamma^{-1}=\left(\begin{array}{ccccc}
2 E & 0 & 0 & \ldots & 0 \\
0 & F & 0 & & \cdot \\
0 & 0 & E & & \cdot \\
& & & & \cdot \\
\cdot & & & & \\
\cdot & & & & \\
\cdot & & & & \\
0 & \ldots & & & 2 E
\end{array}\right)
$$

and the successive terms of (A36) diminish as powers of $G E$ and $G F$, which are small compared to unity. Hence the first term of (A36) is sufficient for our purposes, for use in (A35):

$$
\begin{aligned}
X \doteq & \frac{E}{n}\left(\frac{4 a_{1}}{A}-\frac{2 g_{1}}{G}, 0, \frac{2 a_{3}}{A}-\frac{g_{2}+g_{3}}{G}, 0 \ldots\right) \\
& -\frac{F}{n}\left(0, \frac{2 d_{2}}{D}-\frac{g_{1}+g_{2}}{G}, 0, \frac{2 d_{4}}{D}-\frac{g_{3}+g_{4}}{G}, 0 \ldots\right)
\end{aligned}
$$

and

$$
\begin{aligned}
R \doteq \frac{1}{n G}+ & \frac{E}{n^{2}}\left\{2\left(\frac{2 a_{1}}{A}-\frac{g_{1}}{G}\right)\left(\frac{2 b_{1}}{B}-\frac{g_{1}}{G}\right)\right. \\
& \left.+\left(\frac{2 a_{3}}{A}-\frac{g_{2}+g_{3}}{G}\right)\left(\frac{2 b_{3}}{B}-\frac{g_{2}+g_{3}}{G}\right)+\ldots\right\} \\
& +\frac{F}{n^{2}}\left\{\left(\frac{2 c_{2}}{C}-\frac{g_{1}+g_{2}}{G}\right)\left(\frac{2 d_{2}}{D}-\frac{g_{1}+g_{2}}{G}\right)\right. \\
+ & \left.\left(\frac{2 c_{4}}{C}-\frac{g_{3}+g_{4}}{G}\right)\left(\frac{2 d_{4}}{D}-\frac{g_{3}+g_{4}}{G}\right)+\ldots\right\} .
\end{aligned}
$$




\section{Appendix B}

A power series expansion yields

$$
\begin{aligned}
\left(\Gamma+\gamma_{0}\right)^{-1} & \equiv(\Gamma+G S)^{-1}=\Gamma^{-1}\left(I+G S \Gamma^{-1}\right)^{-1} \\
& =\Gamma^{-1}\left(I-G S \Gamma^{-1}+G^{2} S \Gamma^{-1} S \Gamma^{-1}-\ldots\right) \\
& =\Gamma^{-1}-G \Gamma^{-1} S \Gamma^{-1}+G^{2} \Gamma^{-1} S \Gamma^{-1} S \Gamma^{-1}-\ldots
\end{aligned}
$$

The series converges if the norm of $S \Gamma^{-1}$ is less than $1 / G$. A sufficient condition is

$$
G<\min \left(\frac{\sqrt{2}}{4}(A+B), \frac{C+D}{2}\right) .
$$

Premultiplication of the series by $\beta_{0}$ yields: $\beta_{0}\left(\Gamma+\gamma_{0}\right)^{-1}=\beta_{0} \Gamma^{-1}-G \beta_{0} \Gamma^{-1}\left(S \Gamma^{-1}\right)$

$$
+G^{2} \beta_{0} \Gamma^{-1}\left(S \Gamma^{-1}\right)^{2}-\ldots .
$$

For convenience, let

$$
E \equiv \frac{1}{A+B}, F \equiv \frac{1}{C+D}
$$

then

$$
\begin{aligned}
& \Gamma^{-1}=\left(\begin{array}{ccccc}
2 E & 0 & 0 & \ldots & 0 \\
0 & F & 0 & & \vdots \\
0 & 0 & E & & \\
\vdots & & & & \\
0 & \ldots & & & 2 E
\end{array}\right) \\
& \beta_{0} \Gamma^{-1}=\left(\begin{array}{rrrll}
-A E & 0 & -A E & 0 & \ldots \\
-B E & 0 & -B E & 0 & \ldots \\
0 & -C F & 0 & -C F & \ldots
\end{array}\right) \\
& S \Gamma^{-1}=\left(\begin{array}{rrrrrr}
2 E & -F & 0 & 0 & \ldots & 0 \\
-2 E & 2 F & -E & 0 & & \vdots \\
0 & -F & 2 E & -F & & \\
0 & 0 & -E & 2 F & \\
\vdots & \vdots & & & & \\
0 & \ldots & & & & 2 E
\end{array}\right) .
\end{aligned}
$$

We compute

$$
\beta_{0} \Gamma^{-1}\left(S \Gamma^{-1}\right)=\left(\begin{array}{rrrr}
-2 A E^{2} & 2 A E F & -2 A E^{2} & \ldots \\
-2 B E^{2} & 2 B E F & -2 B E^{2} & \ldots \\
2 C E F & -2 C F^{2} & 2 C E F & \ldots
\end{array}\right)
$$

and

$$
\beta_{0} \Gamma^{-1}\left(S \Gamma^{-1}\right)^{2}=\left(\begin{array}{cccc}
-4 A E^{2}(E+F) & 4 A E F(E+F) & -4 A E^{2}(E+F) & \ldots \\
-4 B E^{2}(E+F) & 4 B E F(E+F) & -4 B E^{2}(E+F) & \ldots \\
4 C E F(E+F) & -4 C F^{2}(E+F) & 4 C E F(E+F) & \ldots
\end{array}\right) .
$$

Comparison of (B9) with (B8) shows that

$$
\beta_{0} \Gamma^{-1}\left(S \Gamma^{-1}\right)^{2}=2\left(E+F^{\prime}\right) \beta_{0} \Gamma^{-1}\left(S \Gamma^{-1}\right)
$$

so that

$$
\beta_{0} \Gamma^{-1}\left(S \Gamma^{-1}\right)^{k+1}=2^{k}(E+F)^{k} \beta_{0} \Gamma^{-1} S \Gamma^{-1}
$$

allowing (B3) to be evaluated as

$$
\begin{aligned}
\beta_{0}\left(\Gamma+\gamma_{0}\right)^{-1}= & \beta_{0} \Gamma^{-1}-G \beta_{0} \Gamma^{-1} S \Gamma^{-1} \\
& \left\{1-2 G(E+F)+4 G^{2}(E+F)^{2}-\ldots\right\} \\
= & \beta_{0} \Gamma^{-1}-\frac{G}{1+2 G(E+F)} \beta_{0} \Gamma^{-1} S \Gamma^{-1} . \quad(\mathrm{B} 12)
\end{aligned}
$$

We note that the result (B12) is valid (by analytic continuation) without restriction on the size of $G$. Substituting (B6) and (B8) into (B12) yields

$\beta_{0}\left(\Gamma+\gamma_{0}\right)^{-1}=-[1+2 G(E+F)]^{-1}$

$$
\begin{aligned}
& \left\{2 G E F\left(\begin{array}{ccc}
A & A & A \ldots \\
B & B & B \ldots \\
C & C & C \ldots
\end{array}\right)\right. \\
& \left.+\left\{\begin{array}{ccccc}
A E & 0 & A E & 0 & \ldots \\
B E & 0 & B E & 0 & \ldots \\
0 & C F & 0 & C F & \ldots
\end{array}\right)\right\} \text {. }
\end{aligned}
$$

(Paper 69C3-197) 